\title{
Protective Effects of Tribulus terrestris, Avena sativa, and White Ginseng Powder on Bone Mineral Density in Hypercholesterolemic Rats
}

\author{
Mehmet Akdoğan ${ }^{1}$, Huri Tilla İlçe ${ }^{2}$, Ali Bilgilii ${ }^{3}$ and Başak Hanedan ${ }^{4}$ \\ 1. Department of Medical Biochemistry, Faculty of Medicine, Sakarya University, Sakarya 54050, Turkey \\ 2. Department of Nuclear Medicine, Faculty of Medicine, Sakarya University, Sakarya 54050, Turkey \\ 3. Department of Pharmacology and Toxicology, Faculty of Veterinary Medicine, Ankara University, Ankara 06110, Turkey \\ 4. Department of Internal Medicine, Faculty of Veterinary Medicine, Ataturk University, Erzurum 25240, Turkey
}

\begin{abstract}
This study investigated the effects of the herbal compounds Tribulus terrestris (TT), Avena sativa (AS), white ginseng (WG), and a triple combination (TC) powder on the serum total cholesterol, low-density lipoprotein cholesterol (LDL-C), high-density lipoprotein cholesterol (HDL-C), triglyceride, and the tibial bone mineral density (BMD) levels in rats fed a high-cholesterol diet. This study comprised Group I (normal pellet feed), Group II (pellet feed with 2\% cholesterol), Group III (2\% cholesterol plus 15\% TT), Group IV (2\% cholesterol plus 7.5\% AS), Group V (2\% cholesterol plus 5\% WG) and Group VI (2\% cholesterol plus 7.5 TT\% + 3.75\% AS, 2.5\% WG). The serum total cholesterol, LDL-C, HDL-C, triglyceride, and tibial BMD $\left(\mathrm{g} / \mathrm{cm}^{2}\right)$ levels were measured. Significant decrease in the serum HDL-C levels in Group II than Group I, and significant increase in the serum LDL-C levels in Group II than Groups I and VI were determined. The tibial BMD levels were significantly lower in Groups II and IV than Group I. It was determined that WG and TC significantly prevented the serum total cholesterol increase; TC significantly prevented the serum LDL-C increase; and TT, WG, and TC were non-significantly effective in the improvement of tibial BMD levels.
\end{abstract}

Key words: Lipid and lipoproteins, Tribulus terrestris, Avena sativa, white ginseng, bone mineral density.

\section{Introduction}

Bone mineral density (BMD) is maintained via the proper functioning of osteoblast and osteoclast cells [1]. The disruption of physiological bone remodeling leads to osteoporosis [2], with the loss of BMD serving as a major risk factor for both osteoporosis and osteoporotic fracture [3]. Reduction of BMD is an important public health concern in aging populations, particularly in females ranging from 51 to 55 years old and males from 61 to 65 years old [4]. Hypercholesterolemia hastens annual bone loss in postmenopausal women [5] and is associated with osteoporosis [6]. A high-cholesterol diet in rats is associated with an osteoporosis risk, which is possibly caused by the

Corresponding author: Ali Bilgili, Prof. Dr., research fields: pharmacology, toxicology and herbal medicine. inhibition of osteoblast proliferation and differentiation [7]. High-density lipoprotein (HDL) has been shown to have preventative effects against the oxidized low-density lipoprotein cholesterol (LDL-C)-induced apoptosis of osteoblastic cells [8].

Fatty acid esters of estrogen are a component of HDL [9]. Estrogen plays an important role in bone health, and the reduction of estrogen related to age is associated with a loss of bone mass [10]. Postmenopausal women not taking hormone replacement therapy compared to those taking hormone replacement therapy have high total cholesterol and LDL-C levels with low BMD levels in the lumbar spine [11]. In addition, testosterone levels have an important role in bone health via the stimulation of bone formation. The reduction of testosterone related to age in men is associated with 
low HDL-C levels [12].

Pharmacological agents are available for osteoporosis treatment that reduce the risk of fractures and correct improper bone resorption and bone remodeling, and these agents include hormones, bisphosphonates, a monoclonal antibody to the receptor activator of nuclear factor kappa-B ligand [13], and statins [14]. However, significant side effects of these pharmacological agents have been demonstrated. For example, atypical femoral fractures can occur after bisphosphonate use [15]; there is a high lung cancer risk with alendronate [16]; bone loss can be seen after the discontinuation of denosumab [17]; there is an increased risk of endometrial cancer with hormone replacement treatment [18], a risk of back pain with teriparatide treatment [19], and a myocardial infarction risk with strontium ranelate [20]; and liver damage can occur with statins [21]. Herbal medicine is recommended as a complement to well-established pharmacological treatments to prevent or treat bone loss [2]. Medicinal plants are known to have an important impact on human health throughout in the world [22].

Tribulus terrestris (TT), belonging to Zygophyllaceae family, is found in the Mediterranean and subtropical regions in the word. Its fruits and roots have been used as an herbal medicine for various diseases. Its pharmacologic active constituents are flavonoids, flavonol glycosides, steroidal saponins, and alkaloids [23]. Its therapeutic effects include acting as an aphrodisiac [24], hypolipidemic [25], hepatoprotector [26], and anti-inflammatory [27].

American ginseng and Korean ginseng have been used in the treatment of many diseases for thousands of years [22]. The active ingredients in ginseng, ginsenosides, are reported to have antioxidant effects [28], anti-adipocyte effects [29], estrogen-like activity [30], and osteogenic activity [31].

Avena sativa (AS) is an oat, or annual grass, that is grown worldwide. It is made up of carbohydrates, proteins, tocols, lipids, alkaloids, flavonoids, saponins, and sterols [32]. It has been shown to have a cholesterol-lowering effect [33], antioxidant activity [34], and antidiabetic activity [35].

There is a great need for elucidating herbal alternative treatments for the improvement of BMD. Thus, the effects of TT, AS, white ginseng (WG), and a triple combination (TC) powder were explored on the serum total cholesterol, LDL-C, HDL-C, triglyceride, and tibial BMD levels in rats fed a high-cholesterol diet.

\section{Material and Methods}

\subsection{Animals and Study Design}

The animals used in this study were 42 male Wistar albino rats, 2.5 months old with a body weight between 200-210 g. The rats were maintained in an experimental room under standard conditions (12 h light/dark cycle; temperature: $25^{\circ} \mathrm{C}$ ) and were adapted to environment for 15 days prior to the start of the experiment. Kobay's Local Ethics Committee approved this experimental procedure.

In this study, the rats were divided into six groups. Group I was fed with normal pellet feed and Group II was fed pellet feed with $2 \%$ added cholesterol. Groups III, IV, V, and VI were fed with $2 \%$ cholesterol plus $15 \%$ TT; $2 \%$ cholesterol plus 7.5\% AS; 2\% cholesterol plus 5\% WG; and 2\% cholesterol plus 7.5 TT\%, 3.75\% AS, 2.5\% WG, respectively. Under anesthesia with xylazine $(10 \mathrm{mg} / \mathrm{kg})$ and ketamine $(90 \mathrm{mg} / \mathrm{kg})$, blood samples and tibial bone tissue samples were obtained. After centrifugation at 3,000 rpm for 10 minutes, sera samples were frozen until biochemical measurements could be performed. At the end of the 90-day study period, the rats were sacrificed.

\subsection{Materials}

Five groups of rats (Groups II, III, IV, V, and VI) were fed pellet feed with $2 \%$ cholesterol (Sigma Aldrich C-75209). The pellet feed was mixed with milled 15\% TT seed (Group III), 7.5\% AS seed (Group IV), and 5\% WG root (Group V). The pellet feed in the 
TC Group (Group VI) included the milled 7.5\% TT seed, 3.75\% AS seed, and 2.5\% WG root.

\subsection{Measurement of the Serum Biochemical Parameters}

The serum total cholesterol, LDL-C, HDL-C, and triglyceride levels were measured using a COBAS-C501 chemistry analyzer (Roche Diagnostics GmbH, D-68298 Mannheim, Germany).

\subsection{Measurement of the Bone Mineral Density}

The tibial BMD $\left(\mathrm{g} / \mathrm{cm}^{2}\right)$ levels in rats were measured using a DEXA scanner (Norland XR-46 bone densitometer, Norland Corp., Fort Atkinson, Wisconsin, USA) with a small-animal scanning software (available from Norland) with a densitometric method.

\subsection{Statistical Analyses}

Statistical analyses were performed using the SPSS 20.0 program. Normal distribution parameters were determined according to the Shapiro-Wilk test. Non-normally distributed and normally distributed parameters were analyzed using the Kruskal-Wallis test and a one-way ANOVA, respectively. The significance between the groups after the one-way ANOVA analysis was determined with Tukey's post hoc test. $p<0.05$ was considered significant.

\section{Results}

\subsection{Assessment of Serum Lipids and Lipoproteins}

The serum total cholesterol level significantly increased in Group II compared to Group I ( $p<0.05$ ). The serum total cholesterol level significantly decreased in Groups V, and VI compared to Group II ( $p$ $<0.05$ ). There were no significant differences among all groups in the serum triglyceride levels $(p>0.05)$. The serum HDL-C level significantly decreased in Group II compared to Group I ( $p<0.05)$. There were no significant differences among Groups I, III, IV, V, and VI for the serum HDL-C levels $(p>0.05)$. The serum LDL-C level significantly increased in Group II in comparison to Group I. In Group VI, the serum LDL-C level significantly decreased in comparison to Group II. All of this information is summarized in Table 1.

\subsection{Assessment of Bone Mineral Density}

The tibial BMD level in Group II significantly decreased compared to Group I $(p<0.05)$. In Group IV, despite AS treatment, the tibial BMD level also significantly decreased compared to Group I. In Groups III, V, and VI, no significant increases were determined as compared to Group II, as shown in Table 1.

Table 1 The effects of TT, AS, WG and TC on total cholesterol, triglyceride, HDL-C, LDL-C, and BMD levels in rats fed a high-cholesterol diet.

\begin{tabular}{lllllll}
\hline Parameters & Group I & Group II & Group III & Group IV & Group V & Group VI \\
\cline { 2 - 7 } & Control & 2\% Chol & 2\% Chol + TT & 2\% Chol + AS & 2\% Chol + WG & 2\% Chol + TC \\
\hline $\begin{array}{l}\text { Total cholesterol } \\
(\mathrm{mg} / \mathrm{dL})\end{array}$ & $71(56-85)$ & $89(84-107)^{\mathrm{a}}$ & $74(68-112)$ & $74(67-80)$ & $68(57-89)^{\mathrm{b}}$ & $71(57-75)^{\mathrm{c}}$ \\
$\begin{array}{l}\text { Triglyceride } \\
(\mathrm{mg} / \mathrm{dL})\end{array}$ & $56.86 \pm 4.67$ & $62.00 \pm 8.18$ & $53.86 \pm 4.38$ & $53.29 \pm 3.13$ & $51.57 \pm 6.56$ & $49.14 \pm 2.65$ \\
HDL-C (mg/dL) & $41.14 \pm 2.63$ & $30.71 \pm 1.68^{\mathrm{d}}$ & $33.00 \pm 3.03$ & $35.43 \pm 1.47$ & $37.29 \pm 1.40$ & $38.14 \pm 0.63$ \\
LDL-C (mg/dL) & $32(29-41)$ & $39(37-43)^{\mathrm{e}}$ & $37(34-43)$ & $37(31-39)$ & $33(30-41)$ & $32(29-37)^{\mathrm{f}}$ \\
BMD (g/cm $\left.{ }^{2}\right)$ & $0.1248 \pm 0.0033$ & $0.1080 \pm 0.0034^{\mathrm{g}}$ & $0.1161 \pm 0.0026$ & $0.1123 \pm 0.0033^{\mathrm{h}}$ & $0.1169 \pm 0.0019$ & $0.1187 \pm 0.0019$ \\
\hline
\end{tabular}

Group I: Control group; Group II: Cholesterol-treated group; Group III: 2\% Cholesterol + TT-treated group; Group IV: 2\% Cholesterol + AS-treated group; Group V: 2\% Cholesterol + WG-treated group; Group VI: 2\% Cholesterol + TC-treated group.

Each group consisted of 7 rats. All values were presented as mean \pm SEM or median (minimum - maximum).

${ }^{\mathrm{a}} p<0.05$ versus Group I; ${ }^{\mathrm{b}} p<0.05$ versus Group II; ${ }^{\mathrm{c}} p<0.05$ versus Group II; ${ }^{\mathrm{d}} p<0.05$ versus Group I; ${ }^{\mathrm{e}} p<0.05$ versus Group I; ${ }^{\mathrm{f}}$ $p<0.05$ versus Group II; ${ }^{\mathrm{g}} p<0.05$ versus Group I; ${ }^{\mathrm{h}} p<0.05$ versus Group I. 


\section{Discussion}

Bone cells are susceptible to disturbances in cholesterol homeostasis due to poor cholesterol efflux and alterations in serum cholesterol levels, resulting in changes in the maturation, differentiation, and stabilization of bone [6]. Several studies have indicated that a high-cholesterol diet in rats causes the increase of total cholesterol and LDL-C levels and the decrease of HDL-C levels [29, 36]. Similarly, in this study, a high-cholesterol diet in rats caused an increase in the serum total cholesterol and LDL-C levels and a decrease in the serum HDL-C levels. This suggests that a high-cholesterol diet leads to an increase in the harmful cholesterol levels in the body.

Lipids have been demonstrated to accumulate in the bones of mice [37]. This lipid accumulation in the bone vessel sub-endothelial matrix, inhibiting the differentiation of osteoblast cells and oxidation of lipids, would be anticipated to induce the differentiation of osteoclast precursor cells [38].

The relationship between hypercholesterolemia and BMD has been revealed in some studies [5-7], but not all studies confirm these results [39, 40]. High serum cholesterol levels have been shown to hasten annual bone loss in women after menopause [5]. Furthermore, dyslipidemia has been suggested to trigger osteoporosis, as evidenced by high-cholesterol and low BMD levels in premenopausal women [41]. Similarly, it has been suggested that a low BMD level is related to dyslipidemia in South Korean men [42]. Hypercholesterolemia has been reported to cause bone resorption via osteoclastogenesis in mice [6]. Thus, in this study, rats were fed a high-cholesterol diet in order to study the association between hypercholesterolemia and BMD. Decreased tibial BMD and increased serum total cholesterol and LDL-C levels were found in rats of Group II fed a high-cholesterol diet. This suggests that hypercholesterolemia can lead to BMD loss in rats via the possible mechanism of osteoblast cell inhibition.
The mechanism of BMD loss due to a high-cholesterol diet in rats has been explained by You et al. [7] using decreased serum ALP and osteocalcin levels and increased carboxy-terminal collagen crosslink levels. In addition, You et al. [7] have demonstrated an increase in gene expression related to bone resorption and a decrease in gene expression related to bone formation in rats fed a high-cholesterol diet.

Hypercholesterolemia is an important public health concern in both women and men because of its association with BMD. Therapeutic agents are commercially available to treat osteoporosis and hypercholesterolemia, but these agents often have adverse effects in humans. Therefore, medicinal herbs are being highly researched to treat these diseases. In this study, the effects of the medicinal plants TT, AS, and WG were studied on hypercholesterolemia and BMD in rats fed a high-cholesterol diet.

Research on ginseng, because it is safe and inexpensive, has been commonly carried out for the treatment of various diseases. Black ginseng has been reported to significantly decrease serum total cholesterol and LDL-C levels and attenuate the expression levels of cholesterol-forming and cholesterol-storing genes in rats fed a high-cholesterol diet [36]. Consistent with the above study results, this study revealed that WG treatment could significantly decrease the serum total cholesterol levels in rats fed a high-cholesterol diet, though there were no changes in the serum HDL-C and triglyceride levels. This suggests that WG has hypocholesterolemic effects on rats.

Moreover, investigations have recently focused on the osteogenic effects of ginseng saponins [31]. Panax notoginseng saponins have been demonstrated to encourage the osteogenic differentiation of the bone marrow stromal cells of rats by stimulating ALP, core-binding factor a1 (Cbfa1), osteocalcin (OC), bone sialoprotein (BSP) expression, and inhibiting peroxisome proliferator activated receptor gamma 2 (PPAR $\gamma 2)$ expression in a dose-dependent manner [31]. 
Huang et al. [28] revealed that ginsenoside- $R b_{2}$ decreases bone-resorbing cytokines, such as the receptor activator of nuclear factor kappa-B ligand (RANKL) and IL-6 induced by oxidative stress in MC3T3-E1 cells, and increases BMD in ovariectomized mice. In addition, Panax notoginseng saponins decreased bone marrow adipogenesis and prevented bone mass loss as well as decreased the serum activities of the C-terminal telopeptides of type I collagen, a bone resorption marker, when used to counteract bone loss induced by ovariectomy in rats [43]. In this study, significant BMD loss was achieved due to hypercholesterolemia in rats. Though non-significant, WG could somewhat prevent the tibial BMD loss via the possible inhibition of bone marrow adipogenesis by decreasing the total cholesterol and LDL-C levels in rats with hypercholesterolemia.

TT is commonly used as medicinal herb in the treatment of various diseases in many countries. The induction of bone formation due to an increase in testosterone production [12] and hypolipidemic activities [25, 44] have been reported as consequences of TT treatment in several studies. However, its effect is not known on BMD in cases caused by hypercholesterolemia. In this study, non-significant decreases in the total cholesterol levels were found in rats with hypercholesterolemia which were treated with TT. In addition, TT showed non-significant increases in BMD in rats with hypercholesterolemia. Non-significant improvements in BMD might be attributable to the slight cholesterol-lowering effect of TT and its possible testosterone-increasing effect.

AS is also known as oat, which is cultivated worldwide. Oat is traditionally used as an herbal remedy because of its anti-oxidant, anti-inflammatory, and anti-cholesterolemic activities, among others [32]. An oat diet has been demonstrated to have hypocholesterolemic activities by aiding in the excretion of fecal total lipids, total cholesterol, and bile acids in hamsters [45]. An in vitro study has demonstrated that the oat bioactive compounds, avenanthramides, can act on bone cells by preventing osteoblast/osteocyte apoptosis and increasing osteoclast apoptosis [46]. However, an in vivo study regarding the effects of oat on bone in cases caused by hypercholesterolemia is not currently available. This study demonstrated that oat in the diet can significantly decrease total cholesterol levels. However, its effect on BMD was unsatisfactory as seen from the lower BMD levels in Group IV as compared to Group I. Furthermore, this study revealed that the TC significantly decreased the total cholesterol and LDL-C levels in hypercholesterolemic rats, and non-significantly increased the BMD levels.

\section{Conclusions}

This study revealed that WG and TC had anti-cholesterolemic effects, and TT, WG, and TC were non-significantly effective in the improvement of tibial BMD levels. Further research on TT, AS, and WG active compounds is needed to prevent or treat bone loss.

\section{Declaration of Conflicting Interests}

The author(s) declared no potential conflicts of interest with respect to the research, authorship, and/or publication of this article.

\section{References}

[1] Kogianni, G., and Noble, B. S. 2007. "The Biology of Osteocytes.” Curr Osteoporos Rep 5: 81-6.

[2] Ivanova, S., and Vasileva, L. 2017. "Current and Emerging Strategies in Osteoporosis Management.” Curr Pharm Des Doi: 10.2174/1381612823666170714122714.

[3] Atsumi, K., Kushida, K., Okamoto, S., and Aoshima, H. 2001. "Bone Mineral Density at Various Sites for Prediction of Vertebral Fractures in Hemodialysis Patients.” J Bone Miner Res 16: S544-54.

[4] Cui, R., Zhou, L., Li, Z., Qi, Z., and Zhang, J. 2016. "Assessment Risk of Osteoporosis in Chinese People: Relationship among Body Mass Index, Serum Lipid Profiles, Blood Glucose, and Bone Mineral Density.” Clin Interv Aging 11: 887-95.

[5] Tarakida, A., Lino, K., Abe, K., Taniguchi, R., Higuchi, T., Mizunuma, H., and Nakaji, S. 2011. "Hypercholesterolemia Accelerates Bone Loss in 
Postmenopausal Women.” Climacteric 14: 105-11.

[6] Pelton, K., Krieder, J., Joiner, D., Freeman, M. R., Goldstein, S. A., and Solomon, K. R. 2012. "Hypercholesterolemia Promotes an Osteoporotic Phenotype.” Am J Pathol 181: 928-36.

[7] You, L., Sheng, Z. Y., Tang, C. L., Chen, L., Pan, L., and Chen, J. Y. 2011. "High Cholesterol Diet Increases Osteoporosis Risk via Inhibiting Bone Formation in Rats.” Acta Pharmacologica Sinica 32: 1498-504.

[8] Brodeur, M. R., Brissette, L., Falstrault, L., and Moreau, R. 2008. "HDL3 Reduces the Association and Modulates the Metabolism of Oxidized LDL by Osteoblastic Cells: A Protection against Cell Death.” J Cell Biochem 105: 1374-85.

[9] Tikkanen, M. J., Vihma, V., Jauhiainen, M., Hockerstedt, A., Helisten, H., and Kaamanen, M. 2002. “Lipoprotein-Associated Estrogens." Cardiovasc Res 56: 184-8.

[10] Pietschmann, P., Rauner, M., Sipos, W., and Kerschan-Schindi, K. 2009. "Osteoporosis: An Age-Related and Gender-Specific Disease-A Mini-Review.” Gerontology 55: 3-12.

[11] Makovey, J., Chen, J. S., Hayward, C., Williams, F. M., and Sambrook, P. N. 2009. "Association between Serum Cholesterol and Bone Mineral Density.” Bone 44: 208-13.

[12] Van Pottelbergh, I., Braeckman, L., De Bacquer, D., De Backer, G., and Kaufman, J. M. 2003. "Differential Contribution of Testosterone and Estradiol in the Determination of Cholesterol and Lipoprotein Profile in Healthy Middle-Aged Men.” Atherosclerosis 166: 95-102.

[13] Minisola, S., Cipriani, C., Occhiuto, M., and Pepe, J. 2017. "New Anabolic Therapies for Osteoporosis." Intern Emerg Med Doi: 10.1007/s11739-017-1719-4.

[14] Solomon, D. H., Finkelstein, J. S., Wang, P. S., and Avorn, J. 2005a. "Statin Lipid-Lowering Drugs and Bone Mineral Density.” Pharmacoepidemiol Drug Saf 14: 219-26.

[15] Patel, R. N., Ashraf, A., and Sundaram, M. 2016. “Atypical Fractures Following Bisphosphonate Therapy." Semin Musculoskelet Radiol 20: 376-81.

[16] Chen, L. X., Ning, G. Z., Zhou, Z. R., Li, Y. L., Zhang, D., $\mathrm{Wu}, \mathrm{Q}$. L., et al. 2015. "The Carcinogenicity of Alendronate in Patients with Osteoporosis: Evidence from Cohort Studies." PLoS One 10: e0123080. Doi: 10.137/journal.pone.0123080.

[17] Tsourdi, E., Langdahl, B., Cohen-Solal, M., Aubry-Rozier, B., Eriksen, E. F., Guanabens, N., et al. 2017. "Discontination of Denosumab Therapy for Osteoporosis: A Systematic Review and Position Statement by ECTS.” Bone 105: 11-7. Doi: 10.1016/j.bone.2017.08.003.

[18] Marjoribanks, J., Farguhar, C., Roberts, H., Lethaby, A., and Lee J. 2017. "Long-Term Hormone Therapy for Perimenopausal and Postmenopausal Women.” Cochrane
Database Syst Rev 1: CD004143. Doi: 10.1002/14651858.CD004143.pub5.

[19] Songpatanasilp, T., Mumtaz, M., Chhabra, H., Yu, M., and Sorsaburu, S. 2014. "Back Pain in Patients with Severe Osteoporosis on Teriparatide or Antiresorptives: A Prospective Observational Study in a Multiethnic Population.” Singapore Med J 55: 493-501.

[20] Atteritano, M., Catalano, A., Santoro, D., Lasco, A., and Benvenga, S. 2016. "Effects of Strontium Ranelate on Markers of Cardiovascular Risk in Postmenopausal Osteoporotic Women.” Endocrine 53: 305-12.

[21] Allen, S. C., and Mamotte, C. D. S. 2017. "Pleiotropic and Adverse Effects of Statins-Do Epigenetics Play a Role?” J Pharmacol Exp Ther 362: 319-26.

[22] Siddiqi, M. H., Siddiqi, M. Z., Ahn, S., Kang, S., Kim, Y. J., Sathishkumar, N., et al. 2013. "Ginseng Saponins and the Treatment of Osteoporosis: Mini Literature Review.” $J$ Ginseng Res 37: 261-8.

[23] Chhatre, S., Nesari, T., Somani, G., Kanchan, D., and Sathaye, S. 2014. "Phytopharmacological Overview of Tribulus terrestris.” Pharmacogn Rev 8: 45-51.

[24] Singh, S., Nair, V., and Gupta, Y. K. 2012. "Evaluation of Aphrodisiac Activity of Tribulus terrestris Linn. in Sexually Sluggish Male Albino Rats.” J Pharmacol Pharmacother 3: 43-7.

[25] Khan, S., Kabir, H., Jalees, F., Asif, M., and Naquvi, K. J. 2011. "Antihyperlipidemic Potential of Fruits of Tribulus terrestris Linn.” Int J Biomed Res 2: 98-101.

[26] Kavitha, P., Ramesh, R., Bupesh, G., Stalin, A., and Subramanian, P. 2011. "Hepatoprotective Activity of Tribulus terrestris Extract against Acetaminophen-Induced Toxicity in a Freshwater Fish.” In Vitro Cell Dev Biol Anim 47: 698-706.

[27] Oh, J. S., Baik, S. H., Ahn, E. K., Jeong, W., and Hong, S. S. 2012. "Anti-Inflammatory Activity of Tribulus terrestris in RAW264.7 Cells.” J Immunol 188 (1 Supplement): 54 .

[28] Huang, Q., Gao, B., Jie, Q., Wei, B. Y., Fan, J., Zhang, H. Y., et al. 2014. "Ginsenoside- $\mathrm{Rb}_{2}$ Displays Anti-Osteoporosis Effects through Reducing Oxidative Damage and Bone-Resorbing Cytokines during Osteogenesis.” Bone 66: 306-14.

[29] Chen, G., Li, H., Zhao, Y., Zhu, H., Cai, E., Gao, Y., et al. 2017. "Saponins from Stems and Leaves of Panax Ginseng Prevent Obesity via Regulating Thermogenesis, Lipogenesis and Lipolysis in High-Fat Diet Induced Obese C57BL/6 Mice.” Food Chem Toxicol 106 (Pt A): 393-403.

[30] Chan, R. Y., Chen, W. F., Dong, A., Guo, D., and Wong, M. S. 2002. "Estrogen-Like Activity of Ginsenoside Rg1 Derived from Panax notoginseng.” J Clin Endocrinol Metab 87: 3691-5.

[31] Li, X. D., Wang, J. S., Chang, B., Chen, B., Guo, C., Hou, 
G. Q., et al. 2011. "Panax notoginseng Saponins Promotes Proliferation and Osteogenic Differentiation of Rat Bone Marrow Stromal Cells.” J Ethnopharmacol 134: 268-74.

[32] Singh, R., De, S., and Belkheir, A. 2013. "Avena sativa (Oat), a Potential Neutraceutical and Therapeutic Agent: An Overview.” Crit Rev Food Sci Nutr 53: 126-44.

[33] Whitehead, A., Beck, E. J., Tosh, S., and Wolever, T. M. S. 2014. "Cholesterol-Lowering Effects of Oat $\beta$-Glucan: A Meta-Analysis of Randomized Controlled Trials.” Am J Clin Nutr 100: 1413-21.

[34] Chen, C. Y., Milbury, P. E., Collins, F. W., and Blumberg, J. B. 2007. "Aventhramides are Bioavailable and have Antioxidant Activity in Humans after Acute Consumption of an Enriched Mixture from Oats.” J Nutr 137: 1375-82.

[35] Livesey, G., Taylor, R., Hulsof, T., and Howlett, J. 2008. “Glycemic Response and Health, a Systematic Review and Meta-Analysis: The Database, Study Characteristics, and Macronutrient Intakes.” Am J Clin Nutr 87: 223-36.

[36] Saba, E., Jeon, B. R., Jeong, D. H., Lee, K., Goo, Y. K., Kim, S. H., et al. 2016. "Black Ginseng Extract Ameliorates Hypercholesterolemia in Rats.” J Ginseng Res 40: 160-8.

[37] Rajendran, K. G., Chen, S. Y., Sood, A., Spielvogel, B. F., and Hall, I. H. 1995. "The Antiosteoporotic Activity of Amine-Carboxyboranes in Rodents.” Biomed Pharmacother 49: 131-40.

[38] Parhami, F., Garfinkel, A., and Demer, L. L. 2000. "Role of Lipids in Osteoporosis." Arterioscler Tromb Vasc Biol 20: 2346-8.

[39] Samelson, E. J., Cupples, L. A., Hannan, M. T., Wilson, P. W., Williams, S. A., Vaccarino, V., et al. 2004. "Long-Term Effects of Serum Cholesterol on Bone Mineral Density in Women and Men: The Framingham
Osteoporosis Study.” Bone 34: 557-61.

[40] Solomon, D. H., Avorn, J., Canning, C. F., and Wang, P. S. 2005b. "Lipid Levels and Bone Mineral Density." Am J Med 118: 1414.

[41] Jeong, T. D., Lee, W., Choi, S. E., Kim, J. S., Kim, H. K., Bae, S. J., et al. 2014. "Relationship between Serum Total Cholesterol and Serum Biochemical Bone Turnover Markers in Healthy Pre-and Postmenopausal Women.” Biomed Res Int 398397. Doi: 10.1155/2014/398397.

[42] Kim, Y. H., Nam, G. E., Cho, K. H., Choi, Y. S., Kim, S. M., Han, B. D., et al. "Low Bone Mineral Density Is Associated with Dyslipidemia in South Korean Men: The 2008-2010 Korean National Health and Nutrition Examination Survey.” Endocr J 60: 1179-89.

[43] Fan, J. Z., Wang, Y., Meng, Y., Li, G. W., Chang, S. X., Nian, H., et al. 2015. "Panax Notoginseng Saponins Mitigate Ovariectomy-Induced Bone Loss and Inhibit Marrow Adiposity in Rats.” Menopause 22: 1343-50.

[44] Tuncer, M. A., Yaymaci, B., Sati, L., Cayli, S., Acar, G., Altug, T., et al. 2009. "Influence of Tribulus terrestris Extract on Lipid Profile and Endothelial Structure in Developing Atherosclerotic Lesions in the Aorta of Rabbits on a High-Cholesterol Diet.” Acta Histochemica 111: 488-500.

[45] Tong, L. T., Guo, L., Zhou, X., Qiu, J., Liu, L., Zhong, K., et al. 2016. "Effects of Dietary Oat Proteins on Cholesterol Metabolism of Hypercholesterolaemic Hamsters.” J Sci Food Agric 96: 1396-401.

[46] Pellegrini, G. G., Morales, C. C., Wallace, T. C., Plotkin, L. I., and Bellido, T. 2016. "Avenanthramides Prevent Osteoblast and Osteocyte Apoptosis and Induce Osteoclast Apoptosis In-Vitro in an Nrf2-Independent Manner.” Nutrients 8: 423. 